Yaroslavl State University

Preprint YARU-HE-99/05

hep-ph/9907493

\title{
Plasma influence on the neutrino - electron processes in a strong magnetic field
}

\author{
A.V. Kuznetsov and N.V. Mikheev \\ Division of Theoretical Physics, Department of Physics, \\ Yaroslavl State University, Sovietskaya 14, \\ 150000 Yaroslavl, Russian Federation
}

\begin{abstract}
An influence of the magnetized electron - positron plasma on the absorption and loss of the energy and momentum in a process of neutrino propagation is investigated. A total contribution of all crossed processes, $\nu \rightarrow \nu e^{-} e^{+}, \nu e^{-} \rightarrow \nu e^{-}, \nu e^{+} \rightarrow \nu e^{+}, \nu e^{-} e^{+} \rightarrow \nu$, is found for the first time, which appears not to depend on the chemical potential of electron-positron gas. Relatively simple expressions for the probability and mean losses of the neutrino energy and momentum are obtained, which are suitable for a quantitative analysis.
\end{abstract}

Based on the talks presented at the Xth International Baksan School "Particles and Cosmology", Baksan Valley, Kabardino Balkaria, Russia, April 19-25, 1999 and

the International Workshop "Particles in Astrophysics and Cosmology:

From Theory to Observation", Valencia, Spain, May 3-8, 1999 


\title{
Plasma influence on the neutrino - electron processes in a strong magnetic field
}

\author{
A.V. Kuznetsov and N.V. Mikheev ${ }^{\mathrm{a} *}$ \\ ${ }^{a}$ Division of Theoretical Physics, Department of Physics, \\ Yaroslavl State University, Sovietskaya 14, \\ 150000 Yaroslavl, Russian Federation
}

\section{Strong magnetic fields in astrophysics}

An understanding of the important role of neutrino interactions in astrophysical processes stimulates a constantly growing interest in the neutrino physics in a dense medium [1]. Matter is usually considered as the 'medium'. It should be stressed, that a strong magnetic field can play the role of the active medium. However, the magnetic field influences significantly the quantum processes only in the case when it is strong enough. There exists a natural scale for the field strength which is the so-called critical Schwinger value $B_{e}=m_{e}^{2} / e \simeq 4.41 \cdot 10^{13} \mathrm{G}$ (we use natural units in which $c=\hbar=1$ ).

The fields of such strength are unattainable in a laboratory. However, the astrophysical objects and processes inside them give us unique possibilities for investigations of the particle physics, and of the neutrino physics especially under extreme conditions of a strong magnetic field. A concept of the astrophysically strong magnetic field has been changed in the recent years (see fig. 1). Whereas the magnetic fields with the strength $10^{9} \div 10^{11} \mathrm{G}$ were considered as 'very strong' near thirty years ago, the fields have been observed at the surface of pulsars have appeared to be stronger, of the order of $10^{12} \div 10^{13} \mathrm{G}$. These fields are now treated as the so-called 'old' magnetic fields. The fields at a moment of the cataclysm like a supernova explosion, when a neutron star was born, could be much greater. In the

\footnotetext{
*Supported by INTAS grant N 96-0659 and in part by Russian Foundation for Basic Research Grant N 98-0216694. A.K. thanks the organizers for the hospitality.
}

present view, the magnetic field strength in the astrophysical processes like a supernova explosion or a coalescence of neutron stars could be as high as $\sim 10^{15} \div 10^{17} \mathrm{G}$. The possible existence of such fields both of toroidal [2, 3], and of poloidal [4] types is the subject of wide speculation.

The mechanism of a generation of such strong magnetic field of the toroidal type suggested by Bisnovatyi-Kogan many years ago 20 looks as the most convincing one. Really, a question should be asked of how natural is it to expect an existence of strong magnetic fields in astrophysics?

i) What can be considered as the exotic case: a star with magnetic field or a star without it? It is believed that a star without magnetic field is rather the exotic object. It is also believed that it is more natural for a star to have the primordial magnetic field before the stage of collapse. The primordial field at the level of $100 \mathrm{G}$ readily leads in the compression to the fields $\sim 10^{12} \mathrm{G}$.

ii) What can be considered as more natural case: a rotating star or a non-rotating one? It is believed that a non-rotating star is rather the exotic object.

iii) What kind of collapse looks more exotic: a compression as a solid body or a compression with the gradient of the angular velocity? It is believed that a compression without the gradient is rather the exotic case.

The above three points are necessary for a realization of the Bisnovatyi-Kogan mechanism. The field lines, by virtue of the angular velocity gradient, become twisted and more dense. As the analysis shows, the fields could be winded from $\sim 10^{12} \mathrm{G}$ to $\sim 10^{16} \mathrm{G}$ during a few seconds. 
Figure 1. Evolution of the notion 'strong magnetic field' in astrophysics.

Thus the appearance of strong magnetic fields of the toroidal type $\sim 10^{16} \mathrm{G}$ in the stellar collapse looks more natural than the absense of such fields.

It should be emphasized that the field of the order of $10^{16} \mathrm{G}$ is really a rather dense medium with the mass density

$\rho=\frac{B^{2}}{8 \pi} \simeq 0.4 \cdot 10^{10} \frac{\mathrm{g}}{\mathrm{cm}^{3}} \cdot\left(\frac{B}{10^{16} \mathrm{G}}\right)^{2}$,

which is comparable with the plasma mass density $10^{10}-10^{12} \mathrm{~g} / \mathrm{cm}^{3}$ to be typical for the envelope of an exploding supernova.

\section{Neutrino - electron processes in a strong magnetic field}

Neutrino does not interact with a magnetic field directly, except for models where it has an unusually large magnetic moment. The field influence on a neutrino is provided by quantum processes of its weak interaction with charged fermions. Electron plays here the main role as the particle with the maximal specific charge $e / m_{e}$.

If the momentum transferred is relatively small, $\left|q^{2}\right| \ll m_{W}^{2}$, the weak interaction of neutrinos with electrons could be described in the local limit by the effective Lagrangian of the following form (for additional conditions of applicability of the local limit in a strong field see below)

$\mathcal{L}=\frac{G_{F}}{\sqrt{2}}\left[\bar{e} \gamma_{\alpha}\left(C_{V}-C_{A} \gamma_{5}\right) e\right]\left[\bar{\nu} \gamma^{\alpha}\left(1-\gamma_{5}\right) \nu\right]$,

where $C_{V}= \pm 1 / 2+2 \sin ^{2} \theta_{W}, C_{A}= \pm 1 / 2$. Here the upper signs correspond to the electron neutrino $\left(\nu=\nu_{e}\right)$ when both $Z$ and $W$ boson exchange contributes to the interaction. The lower signs correspond to $\mu$ and $\tau$ neutrinos $(\nu=$ $\left.\nu_{\mu}, \nu_{\tau}\right)$, when the $Z$ boson exchange is only presented in the Lagrangian (2).

Two parameters are essential in the analysis of definite neutrino processes in a magnetic field, which are the field strength and the neutrino energy. Thus, two asymptotic limits exist where calculations become much easier.

i) The 'weak' field limit.

We call the field as a 'weak' one in the case $e B \ll E_{\nu}^{2}$. This condition can be rewritten in the Lorentz invariant form. The presence of the particle four-momentum $p^{\alpha}=(E, \mathbf{p})$ allows, in 
parallel with the field invariant

$e^{2} F_{\mu \nu} F^{\nu \mu} \equiv e^{2}(F F)=-2 e^{2} B^{2}$,

to construct the dynamical invariant

$e^{2} p_{\mu} F^{\mu \nu} F_{\nu \rho} p^{\rho} \equiv e^{2}(p F F p)=e^{2} B^{2} E_{\nu}^{2} \sin ^{2} \theta$,

where $\theta$ is an angle between the particle momentum $\mathbf{p}$ and the magnetic field induction $\mathbf{B}$. The invariant (化) is most commonly used in the dimensionless form

$\chi^{2}=e^{2}(p F F p) / m^{6}$.

Thus the condition of the field 'weakness' appears

$$
\left[e^{2}(F F)\right]^{3 / 2} \ll e^{2}(p F F p) .
$$

In this case the electrons occupy the higher Landau levels. It is well-known that one can use the crossed field approximation, when the invariant $(F F)=0$, in the investigations of neutrino processes in the limit (6).

Returning to the local Lagrangian (2) we could formulate the conditions of its applicability in a strong magnetic field as follows: the $W$ boson mass should exceed essentially the field parameters (3) and (4)

$m_{W}^{2} \gg\left[e^{2}(F F)\right]^{1 / 2},\left[e^{2}(p F F p)\right]^{1 / 3}$,

which yields correspondingly $e B \ll m_{W}^{2}$,

$E \ll m_{W}^{3} / e B$.

ii) The 'strong' field limit.

In this limit the field strength $B$ appears to be the largest physical parameter, $e B \gg E^{2}$, or in the invariant form

$$
\left[e^{2}(F F)\right]^{3 / 2} \gg e^{2}(p F F p) \text {. }
$$

In this case the electrons occupy only the lowest Landau level.

Most likely the first investigation of the neutrino - electron process induced by a magnetic field was performed for the so-called synchrotron emission of neutrino pairs by electrons, $e \rightarrow$ $e \nu \bar{\nu}$ [5] which is kinematically forbidden in vacuum. The crossed process to the neutrino pair emission is the production of the electron positron pair by a neutrino, $\nu \rightarrow \nu e^{-} e^{+}$, which is also kinematically opened only in the presence of a magnetic field. The probability of this process in a 'weak' field was calculated in the papers [6.6]. In our papers [8] the process of the production of the $e^{+} e^{-}$pair by a neutrino was investigated in both cases of the 'weak' (6) and 'strong' (8) field limits. The process probability was calculated and the four-vector connected with the mean values of the neutrino energy and momentum loss was found

$Q^{\alpha}=-E\left(\frac{d E}{d t}, \frac{d \mathbf{p}}{d t}\right)=E \int\left(p-p^{\prime}\right)^{\alpha} d W$,

where $p$ and $p^{\prime}$ are the momenta of the initial and final neutrinos correspondingly, $d W$ is the total differential probability of the process per unit time.

In the two above-mentioned asymptotic limits we obtained the four-vector $Q^{\alpha}$ in the following form

$$
\begin{aligned}
& \text { i) } e B \gg E^{2} \\
& Q^{\alpha}=\frac{G_{F}^{2} e B(p \varphi \varphi p)^{2}}{48 \pi^{3}} \\
& \times\left\{\left(C_{V}^{2}+C_{A}^{2}\right)\left[p^{\alpha}-2(\varphi \varphi p)^{\alpha}\right]+2 C_{V} C_{A}(\tilde{\varphi} p)^{\alpha}\right\},
\end{aligned}
$$

where $\varphi_{\alpha \beta}$ is the dimensionless external field tensor, $\varphi_{\alpha \beta}=F_{\alpha \beta} / B, \tilde{\varphi}_{\alpha \beta}=\frac{1}{2} \varepsilon_{\alpha \beta \rho \sigma} \varphi^{\rho \sigma}$ is the dual tensor;

ii) $e B \ll E^{2}$

$$
\begin{aligned}
& Q^{\alpha}=\frac{7}{16} \frac{G_{F}^{2}\left(C_{V}^{2}+C_{A}^{2}\right)}{27 \pi^{3}} m^{6} \chi^{2} \\
& \times\left[p^{\alpha}(\ln \chi-1.888)-\sqrt{3} \frac{\beta^{2}}{\chi}(\varphi \varphi p)^{\alpha}\right. \\
& \left.-7.465 \frac{C_{V} C_{A}}{C_{V}^{2}+C_{A}^{2}} \frac{\beta}{\chi^{2 / 3}}(\tilde{\varphi} p)^{\alpha}\right],
\end{aligned}
$$

where $\beta=B / B_{e}$.

This four vector defines the energetic and force action on the medium by a neutrino. Really, after integration over the initial neutrino distribution it defines the energy delivered to the unit volume per unit time, and the volume density of the neutrino force acting on the medium.

\section{Neutrino - electron processes in strongly magnetized plasma}

We have discussed up to now the processes in a pure magnetic field of a high intensity. However, 
in the most of astrophysical objects both components of the active medium, a magnetic field and plasma, are likely to be presented. For example, the astrophysical cataclysms are known like a supernova explosion or a coalescence of neutron stars where rather dense electron - positron plasma exists on the periphery, and strong magnetic fields of the toroidal type can be generated, $B \sim 10^{16} \mathrm{G}$, due to the Bisnovatyi-Kogan mechanism. The mass density of such field, see eq. (11), exceeds essentially the density of the electron component of plasma on the periphery, where the total density is of order of $10^{12} \mathrm{~g} / \mathrm{cm}^{3}$. Thus the magnetic field is the dominating factor, $e B \gg T^{2}, \mu^{2}$, where $\mu$ is the chemical potential of electrons, $T$ is the temperature of plasma. The last condition can be formulated more carefully if one compares the energy densities of the magnetic field and the magnetized electron - positron plasma

$\frac{B^{2}}{8 \pi} \gg \frac{\pi^{2}\left(n_{e^{-}}-n_{e^{+}}\right)^{2}}{e B}+\frac{e B T^{2}}{12}$,

On the other hand, rather high neutrino energies are typical for astrophysical processes, $E_{\nu}, T \gg m_{e}$. Thus we shall consider the physical situation when the field strength appears to be the largest parameter, while the electron mass is the smallest one

$e B \gg E_{\nu}^{2}, \mu^{2}, T^{2} \gg m_{e}^{2}$.

All the neutrino - electron processes described by the Lagrangian (2) can be separated into two parts:

i) the processes with the neutrino - antineutrino pair in the initial or in the final state, $e^{-} e^{+} \leftrightarrow \nu \bar{\nu}$, $e \leftrightarrow e \nu \bar{\nu}$;

ii) the processes where the neutrino presents both in the initial and in the final state, $\nu e^{\mp} \leftrightarrow$ $\nu e^{\mp}, \nu \leftrightarrow \nu e^{-} e^{+}$, and the similar processes with the antineutrino.

A simple analysis shows that in the case of a strong field, see (8) and (12), when the electrons occupy only the lowest Landau level, the processes with the neutrino pair are strongly suppressed. Really, the total spin of the neutrino - antineutrino pair in the center-of-mass system is equal to 1 , while the total spin of the electron - positron pair on the lowest Landau level is equal to zero. Thus the process amplitude is strictly zero for the case of massless particles, and it is suppressed in the considered relativistic limit. Furthermore, the process of the synchrotron emission of the neutrino pair, $e \rightarrow e \nu \bar{\nu}$, just as the reverse process, are forbidden by the energy and momentum conservation when the initial and the final electrons are on the lowest Landau level.

Among the other processes which accompany the neutrino propagation in a magnetic field and plasma, the 'canonical' scattering processes $\nu e^{-} \rightarrow \nu e^{-}, \nu e^{+} \rightarrow \nu e^{+}$are possible without the presence of the magnetic field. The analysis of the $\nu e$ scattering in the magnetized plasma was performed in the paper [9]. The results of numerical calculations of the paper [9] show that the magnetic field influence on the total scattering cross-section is insignificant in the range of parameters considered which corresponds in fact to the 'weak' field limit (6).

Taking account of the plasma influence on the indicated 'exotic' process, $\nu \rightarrow \nu e^{-} e^{+}$, is reduced to a simple modification by insertion of the statistical factor for the electron and the positron in the final state. The second 'exotic' process of the absorption of the pair, $\nu e^{-} e^{+} \rightarrow \nu$, becomes possible only in the presence of a magnetic field and plasma simultaneously.

The probability of this process in a unit time has a physical meaning only being integrated over the initial electron and positron states

$W\left(\nu e^{-} e^{+} \rightarrow \nu\right)$
$=\frac{1}{\mathcal{T}} \int|\mathcal{S}|^{2} d \Gamma_{e^{-}} f_{e^{-}} d \Gamma_{e^{+}} f_{e^{+}} d \Gamma_{\nu}^{\prime}\left(1-f_{\nu}^{\prime}\right)$,

where $\mathcal{T}$ is the total interaction time, $d \Gamma$ is the phase-space element of a particle, $f$ is its distribution function, $f_{\nu}^{\prime}=\left[\exp \left(\left(E^{\prime}-\mu_{\nu}\right) / T_{\nu}\right)+1\right]^{-1}$, $\mu_{\nu}, T_{\nu}$ are the chemical potential and the temperature of the neutrino gas, $f_{e \mp}=\left[\exp \left(\left(E_{\mp} \mp\right.\right.\right.$ $\mu) / T)+1]^{-1}, \mu, T$ are the chemical potential and the temperature of the electron-positron gas. The $\mathcal{S}$ matrix element of the transition coinsides with the one for the process $\nu \rightarrow \nu e^{-} e^{+}[\mathbb{8}]$, to the crossing transformation. We do not present here the details of integration over the phase space of particles, which will be published in an extended paper. The result of our calculation of the proba- 
bility (14) can be presented in the form of a single integral

$$
\begin{aligned}
& W\left(\nu e^{-} e^{+} \rightarrow \nu\right)=\frac{G_{F}^{2} e B T^{2} E}{4 \pi^{3}} \\
& \times\left[\left(g_{V}+g_{A}\right)^{2}(1-u)^{2}+\left(g_{V}-g_{A}\right)^{2}(1+u)^{2}\right] \\
& \times \int_{0}^{\infty} \frac{d \xi}{\left(e^{\xi}-1\right)\left(1+e^{-x+\eta_{\nu}-\xi / \tau}\right)} \ln \frac{\cosh \xi+\cosh \eta}{1+\cosh \eta},
\end{aligned}
$$

where $x=E / T_{\nu}, \tau=T_{\nu} / T, \eta_{\nu}=\mu_{\nu} / T_{\nu}$, $\eta=\mu / T, u=\cos \theta, \theta$ is an angle between the initial neutrino momentum $\mathbf{p}$ and the magnetic field induction $\mathbf{B}$.

The probabilities of the remaining crossed processes $\nu e^{-} \rightarrow \nu e^{-}, \nu e^{+} \rightarrow \nu e^{+}, \nu \rightarrow \nu e^{-} e^{+}$ are defined similarly to (14) with the substitution $f_{e^{\mp}} \rightarrow\left(1-f_{e^{ \pm}}\right)$in every transposition of the electron (positron) from the initial state to the final one. For the total probability of the neutrino interaction with magnetized electron-positron plasma

$$
\begin{aligned}
& W(\nu \longrightarrow \nu) \\
& =W\left(\nu \rightarrow \nu e^{-} e^{+}\right)+W\left(\nu e^{-} e^{+} \rightarrow \nu\right)+ \\
& +W\left(\nu e^{-} \rightarrow \nu e^{-}\right)+W\left(\nu e^{+} \rightarrow \nu e^{+}\right),
\end{aligned}
$$

we obtain

$$
\begin{aligned}
& W(\nu \longrightarrow \nu)=\frac{G_{F}^{2} e B T^{2} E}{4 \pi^{3}}\left\{\left(g_{V}+g_{A}\right)^{2}(1-u)^{2}\right. \\
& \times \int_{0}^{x \tau \frac{1+u}{2}} \frac{\xi d \xi}{\left(1-e^{-\xi}\right)\left(1+e^{-x+\eta_{\nu}+\xi / \tau}\right)} \\
& +\left(g_{V}-g_{A}\right)^{2}(1+u)^{2} \\
& \times \int_{0}^{x \tau \frac{1-u}{2}} \frac{\xi d \xi}{\left(1-e^{-\xi}\right)\left(1+e^{-x+\eta_{\nu}+\xi / \tau}\right)} \\
& +\left[\left(g_{V}+g_{A}\right)^{2}(1-u)^{2}+\left(g_{V}-g_{A}\right)^{2}(1+u)^{2}\right] \\
& \left.\times \int_{0}^{\infty} \frac{\xi d \xi}{\left(e^{\xi}-1\right)\left(1+e^{-x+\eta_{\nu}-\xi / \tau}\right)}\right\} .
\end{aligned}
$$

It is interesting to note that the dependence on the electron chemical potential exactly cancelled in the total probability (17), whereas each of the partial probabilities (16) does depend on $\mu$. We do not know a physical underlying reason of this cancellation up to now. Probably, some property of a completeness of the considered set of processes with respect to the electrons manifests itself here.

The probability (17) defines the partial contribution of the considered processes into the neutrino opacity of the medium. The estimation of the neutrino mean free path with respect to the neutrino-electron processes yields

$\lambda_{e}=\frac{1}{W} \simeq 170 \mathrm{~km} \cdot\left(\frac{10^{3} B_{e}}{B}\right)\left(\frac{5 \mathrm{MeV}}{T}\right)^{3}$.

It should be compared with the mean free path caused by the interaction with nuclei, which is evaluated to be of order of $1 \mathrm{~km}$ at the density value $\rho \sim 10^{12} \mathrm{~g} / \mathrm{cm}^{3}$. At first glance the influence of the neutrino-electron reactions on the process of neutrino propagation is negligibly small. However, a mean free path does not exhaust the neutrino physics in a medium. The mean values of the neutrino energy and momentum loss are more essential in astrophysical applications, and especially the asymmetry of the momentum loss, caused by the influence of an external magnetic field. Many attempts were made to calculate such asymmetry due to neutrino-nucleon processes, motivated by the problem of the observed high space velocities of pulsars (see [10] and references therein). As the analysis shows, the contribution of the neutrino-electron processes into the asymmetry could be comparable with the contributions of the neutrino-nucleon processes.

The components of the four-vector of losses $Q^{\alpha}(9)$ should be calculated from the formula

$Q^{\alpha}=E \int q^{\alpha} d W$,

where $q$ is the difference of the momenta of the initial and final neutrinos, $q=p-p^{\prime}, d W$ is the total differential probability of the processes presented in Eq. (16). The result of our calculation of the zeroth and third components (the magnetic field is directed along the third axis) of the fourvector $Q^{\alpha}$ is

$Q_{0,3}=\frac{G_{F}^{2} e B T^{3} E^{2}}{4 \pi^{3}}$ 


$$
\begin{aligned}
& \times\left\{\left(g_{V}+g_{A}\right)^{2}(1-u)^{2}\right. \\
& \times \int_{0}^{x \tau \frac{1+u}{2}} \frac{\xi^{2} d \xi}{\left(1-e^{-\xi}\right)\left(1+e^{-x+\eta_{\nu}+\xi / \tau}\right)} \\
& \pm\left(g_{V}-g_{A}\right)^{2}(1+u)^{2} \\
& \times \int_{0}^{x \tau \frac{1-u}{2}} \frac{\xi^{2} d \xi}{\left(1-e^{-\xi}\right)\left(1+e^{-x+\eta_{\nu}+\xi / \tau}\right)} \\
& -\left[\left(g_{V}+g_{A}\right)^{2}(1-u)^{2} \pm\left(g_{V}-g_{A}\right)^{2}(1+u)^{2}\right] \\
& \left.\times \int_{0}^{\infty} \frac{\xi^{2} d \xi}{\left(e^{\xi}-1\right)\left(1+e^{-x+\eta_{\nu}-\xi / \tau}\right)}\right\} .
\end{aligned}
$$

Our results for the probability and the four-vector of losses obtained in the case of a pure magnetic field [8], are reproduced from Eqs. (17) and (20) in the limit of a rarefied plasma $\left(T, T_{\nu}, \mu_{\nu} \rightarrow 0\right)$.

\section{Possible astrophysical manifestations}

To illustrate the results obtained we estimate the volume density of the neutrino energy loss per unit time, $\dot{\mathcal{E}}$, and the volume density of the neutrino force acting on plasma along the magnetic field, $\mathcal{F}$

$(\dot{\mathcal{E}}, \mathcal{F})=\int d n_{\nu} \frac{1}{E} Q_{0,3}$,

where $d n_{\nu}$ is the neutrino density

$d n_{\nu}=\frac{d^{3} p}{(2 \pi)^{3}} \frac{1}{e^{\frac{E-\mu_{\nu}}{T_{\nu}}}+1}$.

The case $T_{\nu}=T$ corresponds to the neutrino equilibrium distribution. For the values defined in Eq. (21) we obtain

$$
\begin{aligned}
& (\dot{\mathcal{E}}, \mathcal{F})=\left(g_{V}^{2}+g_{A}^{2}, 2 g_{V} g_{A}\right) \frac{G_{F}^{2} e B T_{\nu}^{7}}{3 \pi^{5}} \\
& \times \int_{0}^{\infty} \frac{x^{3} d x}{e^{x-\eta_{\nu}}+1} \int_{0}^{\infty} \frac{y^{2} d y}{1+e^{-x-y+\eta_{\nu}}} \frac{e^{(\tau-1) y}-1}{e^{\tau y}-1} .
\end{aligned}
$$

The formula (23) demonstrates that the neutrino action on plasma tends to zero in a case when the neutrino distribution is the exact equilibrium one, $\tau=T_{\nu} / T \rightarrow 1$. We stress that the origin of the force density $\mathcal{F}$ in Eq. (23) is the interference of the vector and axial-vector couplings in the effective Lagrangian (2), and it appears as the macroscopic manifestation of the parity violation in weak interaction. At first glance, the main contribution into $\mathcal{F}$ seems to arise from the electron neutrinos, because $g_{V}\left(\nu_{e}\right) \gg g_{V}\left(\nu_{\mu, \tau}\right)$. However, as will be shown later, the dominant contribution comes from the muon and tau neutrino and antineutrino.

For the sake of numerical estimations it is convenient to present Eq. (23) in the following form

$$
(\dot{\mathcal{E}}, \mathcal{F}) \simeq \mathcal{A}\left(g_{V}^{2}+g_{A}^{2}, 2 g_{V} g_{A}\right) \varphi\left(\eta_{\nu}\right) \psi(\tau),
$$

where

$$
\begin{aligned}
& \mathcal{A}=\frac{12 G_{F}^{2} e B T^{7}}{\pi^{5}} \\
& =\left(\frac{B}{10^{16} \mathrm{G}}\right)\left(\frac{T}{4 \mathrm{MeV}}\right)^{7}\left\{\begin{array}{l}
0.55 \cdot 10^{20} \frac{\mathrm{dyne}}{\mathrm{cm}^{3}}, \\
1.6 \cdot 10^{30} \frac{\mathrm{erg}}{\mathrm{cm}^{3} \cdot \mathrm{s}}
\end{array}\right. \\
& \varphi\left(\eta_{\nu}\right)=\frac{\eta_{\nu}^{4}}{24}+\frac{\pi^{2} \eta_{\nu}^{2}}{12}+\frac{7 \pi^{4}}{360}+\mathrm{Li}_{4}\left(-e^{-\eta_{\nu}}\right), \\
& \varphi(0)=\frac{7 \pi^{4}}{720} \simeq 0.947, \\
& \psi(\tau)=\frac{\tau^{7}}{6} \int_{0}^{\infty} \frac{y^{2} d y}{e^{\tau y}-1}\left[e^{(\tau-1) y}-1\right], \\
& \left.\psi(\tau)\right|_{\tau \rightarrow 1} \simeq \tau-1,
\end{aligned}
$$

$\mathrm{Li}_{4}(z)$ is the polylogarythm function. The formulas obtained are also valid for the processes with antineutrino due to the $C P$ invariance of the weak interaction.

The neutrino distribution in a supernova envelope is known to deviate from the equilibrium. Neutrinos outgoing from the central part of a star, having a high temperature, enter the periphery region where the strong magnetic field is generated and where the temperature of the electronpositron gas is lower. The spectral temperatures are known to be different for the neutrinos of various types [1], $T_{\nu_{e}}<T_{\bar{\nu}_{e}}<T_{\nu_{\mu, \tau}} \simeq T_{\bar{\nu}_{\mu, \tau}}$. The neutrino action on plasma leads to the establishment of the thermal equilibrium, $\dot{\mathcal{E}}_{t o t}=0$. In an analysis of the equilibrium the contributions into $\dot{\mathcal{E}}_{t o t}$ of all processes of the neutrino interaction 
with matter should be taken into account. The $\beta$ processes $\nu_{e}+n \leftrightarrow e^{-}+p$ are known, see e.g. [1], to dominate in the energy balance. The rate of the plasma heating due to these processes can be presented in the form $\dot{\mathcal{E}}(\beta) \simeq \mathcal{B}\left(T_{\nu_{e}}-T\right) / T$. As indicated above, the $\beta$ process probability is much greater than the one for the neutrino - electron processes, therefore it is natural that $\mathcal{B} \gg \mathcal{A}$, where $\mathcal{A}$ is defined in Eq. (25). Consequently, the plasma temperature has to be established very close from above to the electron neutrino spectral temperature $\left(T \simeq T_{\nu_{e}}, T>T_{\nu_{e}}\right)$. It is important to note that the force density $\mathcal{F} \neq 0$ when $\dot{\mathcal{E}}_{\text {tot }}=0$. Taking for the estimation $T_{\nu_{e}}=4 \mathrm{MeV}$, $T_{\bar{\nu}_{e}}=5 \mathrm{MeV}, T_{\nu_{\mu, \tau}}=T_{\bar{\nu}_{\mu, \tau}}=8 \mathrm{MeV}$, and considering the chemical potentials to be small, we found for the force density

$\mathcal{F} \simeq 3.6 \cdot 10^{20} \frac{\text { dyne }}{\mathrm{cm}^{3}} \cdot\left(\frac{B}{10^{16} \mathrm{G}}\right)$.

Strictly speaking, the angular asymmetry should be also included into the neutrino distribution (22). However, as the analysis shows, it could change our result (28) at no more than $10 \%$.

The force (28) should be compared with the recent calculation of the similar force caused by the $\beta$ processes 111 . Under the same physical conditions our result appears to be of the same sign and is larger than the result of Ref. [11] by a factor of 2 or more. Thus the role of the neutrino - electron processes in a strong magnetic field could appear more essential than the $\beta$ processes. There is good reason to believe that the results obtained could be useful in a detailed theoretical description of the process of supernova explosion.

\section{Acknowledgements}

We are grateful to G. Raffelt and V. Semikoz for helpful discussions.

\section{REFERENCES}

1. G.G. Raffelt, Stars as Laboratories for Fundamental Physics, University of Chicago Press, Chicago, 1996.

2. G.S. Bisnovatyi-Kogan, Physical Questions of a Theory of the Star Evolution, Nauka Publ., Moscow, 1989 (in Russian); G.S. Bisnovatyi-
Kogan and S.G. Moiseenko, Astro. Zh. 69 (1992) 563 [Sov. Astron. 36 (1992) 285]; G.S. Bisnovatyi-Kogan, Astron. Astrophys. Transactions 3 (1993) 287.

3. M. Ruderman, in "Neutron Stars: Theory and Observation", ed. by J. Ventura and D. Pines, Kluwer Academic. Pub., Dordrecht, 1991; G.J. Mathews et al., preprint astroph/9710229 on xxx.lanl.gov.

4. R.C. Duncan and C. Thompson, Astrophys.J., 392 (1992) L9; C. Thompson and R.C. Duncan, Mon.Not.R.Astron.Soc., 275 (1995) 255; M. Bocquet at al., Astron. and Astrophys., 301 (1995) 757.

5. V.N. Baier, V.M. Katkov, Dokl. Akad. nauk SSSR 171 (1966) 313.

6. E.A. Choban, A.N. Ivanov, ZhETF 56 (1969) 194.

7. A.V. Borisov, V.Ch. Zhukovskii, B.A. Lysov, Izv. Vuz. Fiz. 8 (1983) 30 [Sov. Phys. J. 26 (1983) 701]; A.V. Borisov, A.I. Ternov, V.Ch. Zhukovsky, Phys. Lett. B 318 (1993) 489.

8. A.V. Kuznetsov, N.V. Mikheev, Phys. Lett. B 394 (1997) 123; Yad. Fiz. 60 (1997) 2038 [Phys. At. Nucl. 60 (1997) 1865].

9. V.G. Bezchastnov, P. Haensel, Phys. Rev. D 54 (1996) 3706.

10. P. Arras, D. Lai, E-print astro-ph/9811371.

11. A.A. Gvozdev, I.S. Ognev, Pis'ma v ZhETF. 69 (1999) 337 [JETP Lett. 69 (1999) 365]. 


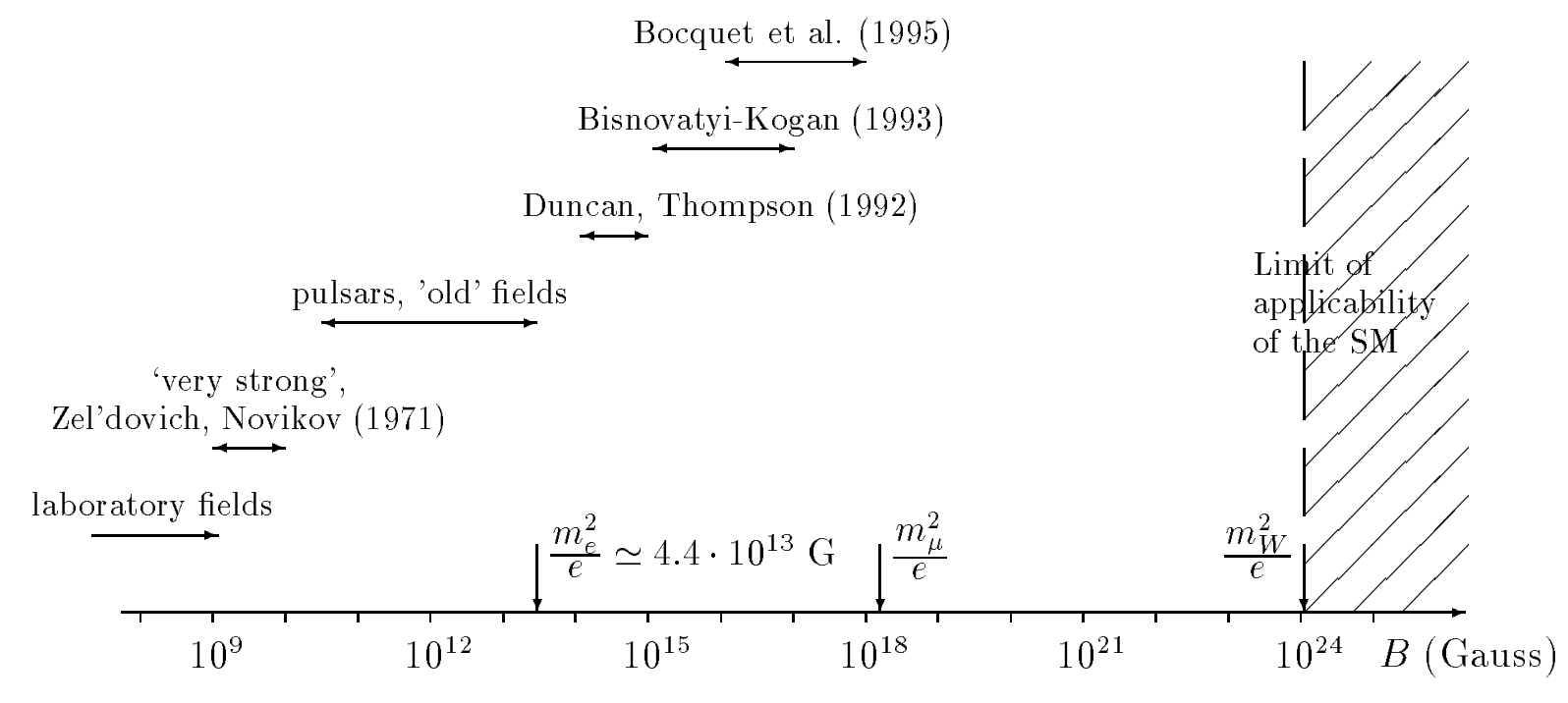

